\title{
O uso do taping no pós-operatório de cirurgia plástica
}

\author{
The use of taping in the postoperative period of plastic surgery \\ El uso de vendaje en el postoperatorio de la cirugía plástica
}

Recebido: 05/11/2021 | Revisado: 09/11/2021 | Aceito: 10/11/2021 | Publicado: 14/11/2021

\author{
Larissa Nunes Correa \\ ORCID: https://orcid.org/0000-0002-5326-2050 \\ Centro Universitário da Amazônia, Brasil \\ E-mail: larissanunes.fisio21@gmail.com \\ Elizane Bentes Sousa \\ ORCID: https://orcid.org/0000-0002-8402-3845 \\ Centro Universitário da Amazônia, Brasil \\ E-mail: elibentes1@gmail.com \\ Naira Patricia Castro de Oliveira \\ ORCID: https://orcid.org/0000-0003-1896-7693 \\ Centro Universitário da Amazônia, Brasil \\ E-mail: nairacastro@outlook.com
}

\begin{abstract}
Resumo
Conceitua que a cirurgia plástica estética (CPE) busca remodelar partes normais do corpo para afins de beleza e autoestima. Por ter apresentado ótimos resultados e pela observação pessoal positiva do profissional na evolução do pós-operatório, a fita atlética vem sendo cada vez mais requisitada pela equipe de cirurgia plástica. Identifica-se como objetivo desse trabalho, expor os resultados apresentados pelo uso do taping no pós-operatório de cirurgia plásticas, bem como os benefícios das suas diversas aplicabilidades para amenizar manifestações da cirurgia. Trata-se de uma revisão narrativa de literatura, buscando uma atualização e meio de obter informações sobre um determinado tema em um curto período de tempo. A pesquisa foi realizada nas seguintes bases de dados: Scientific Electronic Library Online (sciELO), Google Scholar, PUBMED e LILACS (Literatura Latino-Americana e do Caribe em Ciências da Saúde) como também em livros relacionado ao tema proposto. Diante disso, artigos tratam da utilização da bandagem elástica como recurso fisioterapêutico no pós-operatório de cirurgias plásticas, com objetivos comuns como prevenir e tratar o edema, equimose, fibrose e alterações cicatriciais decorrente da cirurgia. Por fim, apesar das bandagens estarem estimulando a recuperação de pacientes pós cirurgia plástica e os resultados parecem ser cada vez mais promissores, é fato que por se tratar de uma técnica nova, há carência de estudos científicos que comprovem o efeito terapêutico causado pela bandagem elástica durante o pós operatório bem como o que questionam alguns bases da técnica.
\end{abstract}

Palavras-chave: Fita atlética; Cuidados pós-operatórios; Cirurgia plástica; Fisioterapia.

\begin{abstract}
Conceptualizes that aesthetic plastic surgery (CPE) seeks to remodel normal parts of the body for beauty and selfesteem. For having presented excellent results and for the positive personal observation of the professional in the evolution of the postoperative period, the athletic tape has been increasingly requested by the plastic surgery team. The objective of this work is to present the results presented by the use of taping in the postoperative period of plastic surgery, as well as the benefits of its various applicability to alleviate manifestations of surgery. It is a narrative literature review, seeking an update and a means of obtaining information on a given topic in a short period of time. The search was carried out in the following databases: Scientific Electronic Library Online (sciELO), Google Scholar, PUBMED and LILACS (Latin American and Caribbean Health Sciences) as well as in books related to the proposed theme. Therefore, articles deal with the use of elastic bandage as a physical therapy resource in the postoperative period of plastic surgery, with common goals such as preventing and treating edema, ecchymosis, fibrosis and scarring changes resulting from the surgery. Finally, although the bandages are stimulating the recovery of patients after plastic surgery and the results seem to be more and more promising, it is a fact that, as this is a new technique, there is a lack of scientific studies that prove the therapeutic effect caused by the elastic bandage during the postoperative period, as well as what question some bases of the technique.
\end{abstract}

Keywords: Athletic tape; Postoperative care; Surgery plastic; Physical therapy.

\section{Resumen}

Conceptualiza que la cirugía plástica estética (CPE) busca remodelar partes normales del cuerpo para la belleza y la autoestima. Por haber presentado excelentes resultados y por la positiva observación personal del profesional en la evolución del postoperatorio, la cinta atlética ha sido cada vez más solicitada por el equipo de cirugía plástica. El 
objetivo de este trabajo es presentar los resultados que presenta el uso del vendaje en el postoperatorio de la cirugía plástica, así como los beneficios de su variada aplicabilidad para paliar las manifestaciones de la cirugía. Es una revisión de la literatura narrativa, que busca una actualización y un medio para obtener información sobre un tema determinado en un período corto de tiempo. La búsqueda se realizó en las siguientes bases de datos: Scientific Electronic Library Online (sciELO), Google Scholar, PUBMED y LILACS (Latin American and Caribbean Health Sciences) así como en libros relacionados con el tema propuesto. Por tanto, los artículos abordan el uso del vendaje elástico como recurso fisioterapéutico en el postoperatorio de la cirugía plástica, con objetivos comunes como prevenir y tratar edemas, equimosis, fibrosis y alteraciones cicatriciales derivadas de la cirugía. Finalmente, aunque los vendajes están estimulando la recuperación de los pacientes tras la cirugía plástica y los resultados parecen ser cada vez más prometedores, es un hecho que, al tratarse de una técnica nueva, faltan estudios científicos que demuestren el efecto terapéutico causado por el vendaje elástico durante el postoperatorio, así como lo que cuestionan algunas bases de la técnica.

Palavras clave: Cinta atlética; Cuidados posoperatorios; Cirugía plástica; Fisioterapia.

\section{Introdução}

A palavra cirurgia plástica é derivada do termo grego "plastikos", que significa forma, suas práticas são datadas desde 4 mil anos a.C. (Carmello, Vinholes \& Feldens, 2013). A cirurgia plástica reconstrutiva tem a finalidade de restaurar alguma parte do corpo que se encontra irregular (Leal et al., 2010). Além disso, para Carmello, Vinholes e Feldens (2013), ela também restabelece a funcionalidade e a forma, deixando mais perto da normalidade prejudicada em decorrência de problemas congênitos ou adquiridos. Em contra partida, Avelar e Viega (2019) conceitua que a cirurgia plástica estética (CPE) busca remodelar partes normais do corpo para afins de beleza e autoestima.

Segundo a pesquisa global mais recente realizado em 2019 pela Sociedade Internacional de Cirurgia Plástica Estética - ISAPS mostra os dois países que mais realizaram procedimentos estéticos. O Brasil é o país que lidera o ranking em procedimento de cirurgia estética com 1.493,673 (13,1\%) deixando os Estados Unidos em segundo lugar com 1.351,917 $(11,9 \%)$, porém, liderando em procedimentos não cirúrgicos, os procedimentos estéticos cirúrgicos mais realizados por brasileiros foram: lipoaspiração $(15,5 \%)$, aumento da mama $(14,1 \%)$, abdominoplastia $(10,4 \%)$, cirurgia da pálpebra $(9,7 \%)$ e aumento de nádegas (7,7\%) (ISAPS, 2019).

Diante desse cenário com o aumento do número de cirurgias plástica no Brasil, também foi surgindo a preocupação com o pré, intra e pós-operatório, com o objetivo de ter um resultado final mais satisfatório com a inclusão de profissionais de saúde que podem atuar na área (Santos et al, 2020). O pós-operatório (PO) de cirurgia inicia quando o paciente desperta da anestesia e seu termino é apenas quando esta totalmente recuperado, e não no momento que recebe alta do hospital (Silva \& Moraes, 2010). A intervenção cirúrgica causa agressão ao tecido podendo alterar a sua funcionalidade, com isso tem se a importância do uso de procedimentos específicos no pós-operatório para reduzir possíveis complicações e reações da cirurgia como o edema, seroma, hematomas, dor, fibrose tecidual, cicatriz e aderência do tecido (Nunes et al., 2020). A fisioterapia dermato funcional tem uma grande importância no período pós-operatório com foco no processo de aceleração da reabilitação, ganhando cada vez mais espaço no campo de atuação da cirurgia plástica por conta de seus recursos fisioterapêuticos utilizados no PO, prevenindo e minimizando possíveis complicações da cirurgia (Migotto \& Simões, 2013).

Kenzo Kase desenvolveu a bandagem elástica como técnica de tratamento enquanto procurava métodos para ajudar no processo de cicatrização de tecidos lesionados resultantes de lesões esportivas (Silva, 2019). Ele foi o responsável por propagar o uso da bandagem elástica. Havendo a necessidade de dar auxilio aos seus pacientes quando os mesmos não estavam em consulta, a princípio usou as bandagens que já existiam até o momento, porém não ficou satisfeito com o resultado. Percebendo que elas dificultavam a amplitude de movimento, comprimiam músculos e articulações e retardavam o processo de cicatrização, foi então que em 1979 produziu sua própria bandagem elástica, intitula Kinesio Tex Tape juntamente com o método Kinesio Taping (Bergesch, Filippin \& Borges, 2017). 
Um dos recursos que vêm sendo utilizado pelo fisioterapeuta no pós-operatório de cirurgia plástica é o Linfotaping, ele é utilizado como auxilio para a drenagem linfática, sendo aplicado seguindo o trajeto do sistema linfático para melhorar o fluxo linfático (Nunes et al., 2020). Porém, para empregar esse método, o profissional deverá possuir treinamento especial envolvendo cuidados para não provocar danos à pele/ou danos referentes à função e a mobilidade do sistema musculoesquelético ligado durante a aplicação, principalmente na etapa do pós-operatório. (Pegorare, Júnior \& Tibola, 2021).

Por ter apresentado ótimos resultados e pela observação pessoal positiva do profissional na evolução do pósoperatório, a bandagem elástica vem sendo cada vez mais requisitada pela equipe de cirurgia plástica. $\mathrm{O}$ fisioterapeuta também vem atingindo uma posição importante no êxito de resultados pós-cirúrgicos. O cenário para a utilização dessa intervenção ainda não está tão esclarecido, porém profissionais que já utilizam a bandagem garantem que ela será uma técnica mais desenvolvida no futuro (Pegorare, Júnior \& Tibola, 2021). Acerca disso, seria considerável averiguar de forma mais metodizada quais os benefícios do uso da bandagem elástica no pós-operatório de cirurgia plástica.

Tendo em consideração o exposto, o objetivo desse estudo é expor os resultados apresentados pelo uso da taping no pós-operatório de cirurgia plástica, bem como os benefícios das suas diversas aplicabilidades para amenizar manifestações da cirurgia.

\section{Metodologia}

O presente estudo trata-se de uma revisão narrativa. Esse tipo de pesquisa em questão traz uma atualização subsequente, visto que o leitor obtém e acrescenta conhecimento em menor tempo sobre um determinado tema. Logo, retrata e debate o desenvolvimento acerca de certa temática (Rother, 2007).

Assim, de inicio, uma questão foi elaborada a de fim descrever estudos publicados relacionados ao uso da bandagem elástica no pós-operatório de cirurgia plástica. 'Quais os beneficios do uso da bandagem elástica no pós-operatório de cirurgia plástica?". Em seguida a definição da pergunta metodológica, selecionaram-se os descritores objetivando retirar artigos não condizem com a proposta da pesquisa ao efetuar a busca nas principais bases de dados. Para esses estudos, a pesquisa em literatura científica se concentrou na coleção Scientific Electronic Library Online (sciELO), Google Scholar, PUBMED e LILACS (Literatura Latino-Americana e do Caribe em Ciências da Saúde) como também em livros relacionado ao tema proposto, e os descritores selecionados foram: fita atlética, cuidados pós-operatório, cirurgia plástica e fisioterapia.

Procurou-se identificar artigos científicos que se encaixassem nos seguintes critérios de inclusão: estudos mais recentes que falassem sobre a aplicação e o resultado do uso da bandagem elástica como recurso terapêutico em pós-operatório de cirurgia plástica, idioma português e Inglês. Foram excluídos artigos que não tratavam de bandagem elástica em pósoperatório de cirurgia plástica, estudos incompletos, artigos duplicados, resumos, resenhas, debates. No total, foram selecionados 10 artigos que se englobavam nos critérios pré-estabelecidos.

\section{Resultados e Discussão}

No Quadro 1 mostra-se 10 artigos de estudos realizados sobre a aplicação do taping no PO de abdominoplastia, lipoaspiração, rinoplastia, bichectomia, mastectomia e cirurgia ortognática. 
Quadro 1 - Exposição de artigos que se relaciona de diferentes formas sobre o uso do taping no pós-operatório de cirurgia plásticas estética atuando tanto no tratamento quanto na prevenção de possíveis reações da cirurgia.

\begin{tabular}{|c|c|c|}
\hline Fontes & Tipo de pesquisa/objetivo & Conclusão \\
\hline Pereira \& Santos, 2016 & $\begin{array}{l}\text { Bibliográfico/ tem como o objetivo relatar a } \\
\text { importância da aplicação do linfotaping no pós- } \\
\text { operatório de abdominoplastia. }\end{array}$ & $\begin{array}{l}\text { Torna-se fundamental a utilização de novos recursos, técnicas } \\
\text { tradicionais, o linfotaping tem sido considerado tão importante } \\
\text { quanto as técnicas já utilizadas, podendo ser uma técnica coadjuvante } \\
\text { de apoio que utiliza a bandagem elástica. }\end{array}$ \\
\hline $\begin{array}{l}\text { Chi, Marquetti \& Dias, } \\
2021\end{array}$ & $\begin{array}{l}\text { Campo/ tem como objetivo avaliar a ocorrência } \\
\text { de equimose de pacientes submetidas à } \\
\text { abdominoplastia e/ou lipoaspiração tradicional de } \\
\text { abdome e flancos, e correlacionar } \\
\text { estatisticamente essas ocorrências com o } \\
\text { tratamento de taping linfático no transoperatório. }\end{array}$ & $\begin{array}{l}\text { O uso do taping linfático do transoperatório de abdominoplastia e } \\
\text { lipoaspiração, reduziu ou anulou a formação de equimose no pós- } \\
\text { operatório, contribuindo para a diminuição do número de } \\
\text { atendimentos fisioterapêuticos, incidência de quadro álgico e } \\
\text { acelerando assim o restabelecimento dos pacientes no pós operatório } \\
\text { das cirurgias de abdominoplastia e/ou lipoaspiração. }\end{array}$ \\
\hline Chi et al, 2016 & $\begin{array}{l}\text { Campo/ tem como objetivo identificar os efeitos } \\
\text { de dois protocolos distintos (DLM associada ao } \\
\text { linfotaping e DLM associada a terapia } \\
\text { combinada e linfotaping) no tratamento da } \\
\text { fibrose secundária ao pós-operatório de } \\
\text { abdominoplastia e lipoaspiração do abdome. }\end{array}$ & $\begin{array}{l}\text { A análise comparativa da avaliação inicial e final, tanto na palpação } \\
\text { quanto na termografia, mostrou que houve redução significativa } \\
\text { (p<0,0001) do quadro fibrótico apresentado pelas pacientes e que os } \\
\text { protocolos foram eficientes no tratamento de fibrose secundarias a } \\
\text { cirurgias de abdominoplastia associada ou não a lipoaspiração. }\end{array}$ \\
\hline Paula, 2017 & $\begin{array}{l}\text { Campo/ tem como objetivo descrever os efeitos } \\
\text { do Puch Tape no tratamento de fibrose e } \\
\text { aderências cicatriciais no pós operatório de } \\
\text { lipoaspiração. }\end{array}$ & $\begin{array}{l}\text { Os resultados apresentados neste estudo de caso sugerem que a } \\
\text { aplicação da bandagem neuromuscular do tipo Puch Tape pode ser } \\
\text { uma alternativa prática, segura e eficaz no tratamento de alterações } \\
\text { cicatriciais. }\end{array}$ \\
\hline Ozucer et al, 2016 & $\begin{array}{l}\text { Campo/ tem como objetivo avaliar o efeito da } \\
\text { bandagem pós-rinoplastia no edema nasal e no } \\
\text { envolvimento nasal }\end{array}$ & $\begin{array}{l}\text { A bandagem pós-rinoplastia ajuda o envelope da pela a se comprimir } \\
\text { á estrutura subjacente e diminuir o edema pós-operatório. O } \\
\text { procedimento pode ser utilizado principalmente em pacientes de pele } \\
\text { grossa, nos quais o drapeamento da pele e o refinamento nasal são } \\
\text { fundamentais para o resultado cirúrgico. }\end{array}$ \\
\hline Morais, 2012 & $\begin{array}{l}\text { Campo/ tem como objetivo verificar os } \\
\text { resultados da aplicação da drenagem linfática } \\
\text { manual (DLM) e das bandas neuromusculares, na } \\
\text { dor, edema, hematoma e pigmentação, do } \\
\text { abdômen e mamas, na reabilitaça após } \\
\text { lipoaspiração para reconstrução mamaria. }\end{array}$ & $\begin{array}{l}\text { A DLM e a aplicação de bandas neuromusculares melhoram } \\
\text { substancialmente aquelas que são as consequências mais comuns no } \\
\text { pós-cirúrgico de lipoaspiração para reconstrução mamária. }\end{array}$ \\
\hline Pelissaro et al, 2021 & $\begin{array}{l}\text { Campo/ tem como objetivo descrever a técnica } \\
\text { cirúrgica da bichectomia com a aplicação da fita } \\
\text { Kinesio para verificar sua eficácia no controle da } \\
\text { formação de edema, com a mensuração em } \\
\text { quatro períodos pós-operatórios. }\end{array}$ & $\begin{array}{l}\text { Após a obtenção dos dados e analise estatística, a aplicação do } \\
\text { kinesio Tape mostrou-se mais eficaz quando comparada ao uso da } \\
\text { crioterapia isolada, no controle do edema pós-operatório em } \\
\text { pacientes submetidos à cirurgia de bichectomia. }\end{array}$ \\
\hline Pekyavas et al, 2014 & $\begin{array}{l}\text { Campo/ tem como objetivo investigar os efeitos } \\
\text { do Kinesio Taping em pacientes com lifedema de } \\
\text { pós mastectomia como terapia descongestiva. }\end{array}$ & $\begin{array}{l}\text { A aplicação do kinesio taping junto com a CDT pode ter um efeito } \\
\text { melhor na diminuição do linfedema, o que pode estimular a redução } \\
\text { do edema para efeitos a longo prazo. }\end{array}$ \\
\hline Chi et al, 2018 & $\begin{array}{l}\text { Campo/ tem como objetivo propor uma } \\
\text { abordagem inédita desde o pré, trans e pós- } \\
\text { operatório para prevenir e minimizar as fibroses, } \\
\text { edema intenso e equimoses, acelerando a } \\
\text { recuperação do paciente e reduzindo o número de } \\
\text { sessões. }\end{array}$ & $\begin{array}{l}\text { Pode-se concluir, com este estudo, que o uso de cosméticos e } \\
\text { nutricosméticos antiglicantes e antiinflamatórios no pré-operatório, } \\
\text { associados à colocação do taping linfático abaixo da espuma de } \\
\text { contenção no transoperatório, reduzem o edema, a formação de } \\
\text { equimose e principalmente a formação de fibrose no pós-operatório. }\end{array}$ \\
\hline Kijak et al, 2018 & $\begin{array}{l}\text { Campo/ tem como objetivo avaliar a eficácia do } \\
\text { método kinesio taping (KT) em pacientes após } \\
\text { cirurgia ortognática na área do crânio facial em } \\
\text { termos de eliminação do inchaço pós-operatório. }\end{array}$ & $\begin{array}{l}\text { A análise do impacto do uso prático do KT linfático nas } \\
\text { complicações após cirurgia ortognática revelou que teve efeito } \\
\text { benéfico na redução do edema. }\end{array}$ \\
\hline
\end{tabular}

Fonte: Correa, Sousa e Oliveira (2021).

As maiorias dos estudos selecionados que estão expostos na tabela trata-se de trabalhos de campos originais como ensaios clínicos, estudos randomizados e adicionalmente uma de produção bibliográfica foi escolhida. Isso comprova que ao longo da pesquisa, as maiorias dos artigos elaboradas sobre a temática são de campo, e exalta o quanto esta sendo importante realizar os estudos voltados para esse recurso comprovando seus benefícios no pós-operatório de cirurgia plástica.

No presente estudo foi possível analisar o efeito do uso do kinesio tape no pós-operatório de cirurgia plástica estéticas como intervenção de possíveis complicações ou reações da cirurgia além da prevenção do mesmo. No intuito de obter resultados positivos sobre o uso do taping na equimose, foi incluído no estudo dois grupos de mulheres como 10 no grupo controle (GC) e 10 no grupo experimental (GE), o GC não recebeu nenhum tipo de tratamento apenas avaliação no pré e pósoperatório, enquanto o GE recebeu o tratamento no trásoperatório e no pós operatório com o uso do taping linfático na região do abdome e flancos. Como resultado do estudo de Chi, Marquetti e Dias (2021), o grupo experimental que recebeu o 
tratamento teve melhor resposta na resolução da equimose em comparação ao grupo controle, além disso, teve também melhora no quadro álgico no GE. Como resposta para o resultado positivo os autores desta pesquisa relataram que o taping provoca um distanciamento da derme e epiderme colaborando para reduzir a cogestão do liquido linfático e explicando a diminuição do edema e da equimose.

Outra complicação comum no PO é a fibrose, cujo é o aumento do deposito tecido fibroso de forma excessiva, ela é percebida após a fase inflamatória e dependendo do tratamento fisioterapêutico pode-se ter bons resultados (Quaresma et al, 2020). Também trás para o paciente varias alterações tanto na redução de mobilidade, sensação de encurtamento, dor e prolonga a recuperação da cirurgia (Pereira et al, 2020). O estudo de Chi et al, (2016) no qual estudou a aplicação do linfotaping com outros recursos fisioterapêuticos sobre a fibrose no pós-operatório de cirurgia plástica de abdômen, mostrou a importância de iniciar o tratamento de forma precoce. Isso porque, o estudo foi realizado com dois grupos dividido de acordo com a fase de reparo tecidual, o grupo da fase proliferativa recebeu como tratamento a drenagem linfática manual e a aplicação do linfotaping, enquanto o grupo da fase de remodelação recebeu como tratamento a DLM associada a terapia combinada (ultrassom e corrente Aussie) e ao linfotaping. Como resultado do estudo ambos os grupos tiveram resultados eficientes no tratamento da fibrose, porém, apenas 1 paciente do grupo de fase de remodelação não presentou a mesma redução do quadro fibrótico em comparação a outros pacientes, pois a mesma iniciou o tratamento após dois anos da cirurgia.

Apesar de não ter muitos estudos voltados para o uso da bandagem neuromuscular para prevenção e tratamento de alterações cicatriciais, esse recurso vem sendo uma estratégica terapêutica na otimização de tensões mecânicas teciduais, a sua aplicação sobre regiões fibróticas no PO de cirurgias plásticas vem promovendo a redução da dor e restabelece a circulação sanguínea e linfática (Lange, 2014). Outra pesquisa realizada por Paula, (2017) tratou-se da utilização da bandagem elástica do tipo Punch tape sobre as regiões fibróticas de nível 3 (detectada em avaliação visual, percebe-se a fibrose com o paciente na posições ereta, decúbito dorsal e ventral), após o tratamento a reavaliação do paciente foi detectado melhora do quadro fibrótico evoluindo para o nível 1 onde apenas é percebida através da palpação.

De forma geral, os artigos tratam da utilização da bandagem elástica como recurso fisioterapêutico no pós-operatório de cirurgias plásticas, com objetivos comuns como prevenir e tratar o edema, equimose, fibrose e alterações cicatriciais decorrente da cirurgia, além de comprovar que esse recurso ajuda no aceleramento da recuperação dos pacientes diminuindo os números de atendimento fisioterapêutico. Também, vale ressaltar que o taping deve ser aplicado por profissionais habilitados com a formação especifica do método, pois para atuar o fisioterapeuta faz uma minuciosa avaliação no tecido e assim usar o método mais adequado para cada caso buscando uma recuperação positiva, eficiente e funcional (Pereira \& Santos, 2016).

\section{Conclusão}

Em suma, a utilização da Fita atlética vem crescente exponencialmente, estando presente em diversos protocolos de tratamento de profissionais. Sua aplicação no pós-operatório de cirurgia plástica vem ganhado espaço, principalmente pelo bom feedback apresentado após o seu uso.

$\mathrm{O}$ uso do taping para a fim de atenuar as manifestações pós-operatórias foram positivos, visto que ouve uma melhora significativa no quadro álgico, na redução ou eliminação de chances para a formação de equimose, auxilia no processo de cicatrização, risco de formação de fibrose e diminuição de linfedema o que por consequência pode promover uma redução do edema em longo prazo, manifestações essas que causam incômodo no paciente. Além do mais, a maioria dos estudos utilizou a bandagem elástica associada a outro método de tratamento.

Apesar das bandagens estarem estimulando a recuperação de pacientes pós-cirurgia plástica e os resultados parecem ser cada vez mais promissores, é fato que por se tratar de um técnica nova, há carência de estudos científicos que comprovem o 
efeito terapêutico causado pela bandagem elástica durante o pós operatório bem como o que questionam alguns bases da técnica.

Portanto, é importante salientar que futuros estudos científicos tragam mais comprovações acerca da real eficácia da bandagem elástica em pós-operatório de cirurgia plástica, investigando como a aplicabilidade dessa técnica age na contenção das manifestações advindas de um PO e seus efeitos benéficos ou não, busquem estabelecer uma relação entre tensão, forma de colocação do taping e seus efeitos fisiológicos, bem como de que forma essa terapêutica pode ser associada a outros métodos de tratamento.

\section{Agradecimentos}

Agradecemos nossa orientadora Naira Patrícia Castro pelo suporte no decorrer do nosso artigo, incentivando, orientando e apoiando essa pesquisa. Somos gratas pela sua dedicação e apoio.

\section{Referências}

Avelar, C., \& Veiga, R. T. (2019). Validação de uma escala sobre propensão feminina a cirurgia plástica estética. Caderno Profissional de MarketingUNIMEP, 7(3), 143-156.

Bergesch, D. P., Filippin, L. I., \& de Jesus Borges, T. (2017). A bandagem elástica adesiva aplicada à estética. SEFIC 2017.

Carmello, F. A., Vinholes, D. B., \& Feldens, V. P. (2013). Avaliação da autoestima no pré-operatório de pacientes submetidas à cirurgia plástica estética em uma clínica privada de Tubarão-SC. Arquivo Catarinense de Medicina, 42(2), 75-80.

Chi, A., de Oliveira, A. V. M., Ruh, A. C., \& Schleder, J. C. (2016). O uso do linfotaping, terapia combinada e drenagem linfática manual sobre a fibrose no pós-operatório de cirurgia plástica de abdome. Fisioterapia Brasil, 17(3), 197-203.

Chi, A., Lange, A., Guimarães, M. V. T. N., \& Santos, C. B. D. (2018). Prevenção e tratamento de equimose, edema e fibrose no pré, trans e pós-operatório de cirurgias plásticas. Rev. bras. cir. plást, 343-354.

Chi, A., Marquetti, M. D. G., \& Dias, M. (2021). Uso do tapinglinfático na prevenção da formação de equimoses em abdominoplastia e lipoaspiração. Revista brasileira de cirurgia plástica, (2), 144-150.

da Silva, A. P., \& de Moraes, M. W. (2010). Incidência de dor no pós-operatório de cirurgia plástica estética.

da Silva, F. A. (2019). Kinesio Taping: aplicação e seus resultados sobre a dor: revisão de literatura. Revista Interdisciplinar Pensamento Científico, 5(2).

de Paula, S. (2017). Punch-tape em fibroses cicatriciais pós-lipoaspiração: um relato de caso. Revista Eletrônica Acervo Saúde, (6), S475-S480.

Dos Santos, N. L., Esteves-De-Oliveira, I. G., Tacani, R. E., Baldan, C. S., Masson, I. F. B., Farcic, T. S., \& Machado, A. F. P (2020) Percepção das pacientes sobre a atuação profissional e os procedimentos realizados no pré, no intra e no pós-operatório de abdominoplastia. Rev. Bras. Cir. Plás. 35 (2):189-197.

Lange, A. (2014). Fisioterapia dermato funcional aplicada à cirurgia plástica: intercorrências, complicações, avaliação e tratamento. Vitória gráfica \& Editora.

Leal, V. C. L. V., Catrib, A. M. F., Amorim, R. F. D., \& Montagner, M. Â. (2010). O corpo, a cirurgia estética e a Saúde Coletiva: um estudo de caso. Ciência \& Saúde Coletiva, 15, 77-86.

Lietz-Kijak, D., Kijak, E., Krajczy, M., Bogacz, K., Łuniewski, J., \& Szczegielniak, J. (2018). The impact of the use of kinesio taping method on the reduction of swelling in patients after orthognathic surgery: a pilot study. Medical science monitor: international medical journal of experimental and clinical research, 24, 3736 .

Migotto, J. S. (2013). Atuação fisioterapêutica dermato funcional no pós-operatório de cirurgias plásticas. Revista Eletrônica Gestão e Saúde, (1), 1646-1658.

Morais, S. C. (2012). O efeito da drenagem linfática manual e das bandas neuromusculares na reabilitação pós-lipoaspiração para reconstrução mamária: estudo de caso (Bachelor's thesis, [sn]).

Nunes, N. D. M. F., de Matos, M. O., Rocha-Lima, A. B. C., Soares, V. C. G., \& Farcic, T. S. (2020). Abordagens terapêuticas utilizadas como ferramenta para restauração funcional e estética no pós-operatório de cirurgia plástica: uma revisão da literatura. Revista eletrônica em Gestão e Tecnologia, 6 (1).

Ozucer, B., Yıldırım, Y. S., Veyseller, B., Tugrul, S., Eren, S. B., Aksoy, F., \& Ozturan, O. (2016). Effect of postrhinoplasty taping on postoperative edema and nasal draping: a randomized clinical trial. JAMA facial plastic surgery, 18(3), 157-163.

Pegorare, A. B. D. S., Oliveira Júnior, S. A. D., \& Tibola, J. (2021). Manual de condutas e práticas em fisioterapia dermatofuncional: atuação no pré e pós operatório de cirurgias plásticas.

Pekyavaş, N. Ö., Tunay, V. B., Akbayrak, T., Kaya, S., \& Karataş, M. (2014). Complex decongestive therapy and taping for patients with postmastectomy lymphedema: a randomized controlled study. European Journal of Oncology Nursing, 18(6), 585-590. 
Research, Society and Development, v. 10, n. 15, e81101522868, 2021

(CC BY 4.0) | ISSN 2525-3409 | DOI: http://dx.doi.org/10.33448/rsd-v10i15.22868

Pelissaro, G. S., da Silva, T. F. R. G., de Souza Herculano, A. B., dos Santos, M. E. S., Oliveira, B. C., da Silva, J. C. L., \& Jardim, E. C. G. (2021). Fita Kinesio para controle de edema após bicectomia: um estudo experimental randomizado. Research, Society and Development, 10 (5), e33610514983e33610514983.

Pereira Sr, S. (2016). Efeitos da aplicação do linfotaping como técnica coadjuvante no pós-operatório cirurgias plásticas abdominais. Revista Visão Universitária, 2(1).

Pereira, D. S., de Almeida Sá, M. L., de Oliveira, J. G., Polese, J. C., \& da Silva, F. S. (2020). Efeito da liberação miofascial em fibrose no pós-operatório de lipoaspiração em abdome: um estudo piloto. Revista interdisciplinar ciências médicas, 4(1), 55-61.

Quaresma, M. R., Santos, T. P. M., de Almeida, C. P., Rodrigues, L. D. C. S., dos Santos, R. M., da Costa, K. A., \& dos Reis Ferreira, T. C. (2020). Recursos fisioterapêuticos no pós-operatório de cirurgia plástica: revisão de literatura. Revista CPAQV-Centro de Pesquisas Avançadas em Qualidade de Vida| 12(2), 2.

Rother, E. T. Revisão sistemática X revisão narrativa. (2007). Editora Técnica da Acta Paulista de Enfermagem.

Sociedade Internacional de Cirurgia Plástica Estética (ISAPS). ISAPS Global Survey Result 2019. https://www.isaps.org/wp-content/uploads/2020/12/GlobalSurvey-2019.pdf.

Sociedade Internacional de Cirurgia Plástica Estética (ISAPS). Pesquisa Global mais recente. https://www.isaps.org/wp-content/uploads/2020/12/ISAPSGlobal-Survey-2019-Press-Release-Portuguese.pdf. 